\title{
Implementing Entropy Codec for H.264 Video Compression Standard
}

\author{
Mohamed Abd Ellatief Elsayed \\ Communication Department, \\ Ain shams university
}

\author{
Abdelhalim Zekry \\ Faculty of Engineering, \\ Ain Shams University
}

\begin{abstract}
Entropy coding is a lossless compression technique which is supported in H.264/AVC standard by different techniques. According to baseline and the extended profiles of H.264/AVC, two variable length techniques are for seen. The first one is context adaptive variable length coding (CAVLC) and the other is exponential Golomb (Exp-Golomb) one. The CAVLC is used to quantize transform residues after reordering them by ZigZag scanning while Exp-Golomb coding is used to quantize other syntax elements. Within the frame of realizing the whole H246 standards, this paper, introduces an implementation of these two codec techniques for baseline profile using Matlab and Simulink. The main concept is to implement CAVLC and Exp-Golomb decoder according to H.264/AVC standard and then device a technique to implement CAVLC \& Exp-Golomb encoder. The different implementations are utilized to verify each other.
\end{abstract}

\section{Keywords}

Context adaptive variable length coder (CAVLC), Entropy coding, Exp-Golomb, H.264/AVC.

\section{INTRODUCTION}

H.264/ MPEG-4 AVC is a video codec standard that is currently one of the most commonly used formats for the recording, compression, and distribution of video content. The standardization of the first version of H.264/AVC was completed in May 2003. The functional block diagram of H.264 encoder/decoder is shown in Fig.1.As shown in Fig.1, entropy coding is the last block in encoder after the transform quantization and the fist block in decoder before the inverse quantization. H.264 supports two techniques for entropy encoding. The first one is the Context adaptive variable length coding (CAVLC) for the quantized transform residues after ordering them by ZigZag scanning together with Exponential Golomb coding, Exp-Golomb, for other syntax elements. The other technique is to use Context adaptive binary arithmetic coding (CABAC).CABAC has a higher compression performance but unlike CAVLC, it is not supported in all H.264 profile like baseline and extended profiles [1].

H.264/ MPEG-4 AVC support Context adaptive variable length coding (CAVLC) as one of entropy encoder methods to achieve a higher compression ratio [2]. Compared with previous variable length coding, CAVLC achieves better coding efficiency. Because of its adaptive part that makes the encoding of each block depend on the number of non zero transform coefficient in the upper left part of the block. But that makes the algorithm complexity higher. CAVLC decoder is used in all the upcoming media applications that have adopted H.264 video standard:

- Digital TV (satellite, cable, IPTV, over-the-air broadcast etc).

\section{- High-Definition Optical Discs (Blu-ray Disc, and HDDVD).}

-Real time Image Processing Applications(video conferencing and video telephony).

-Portable device applications (cell phones and portable media players).

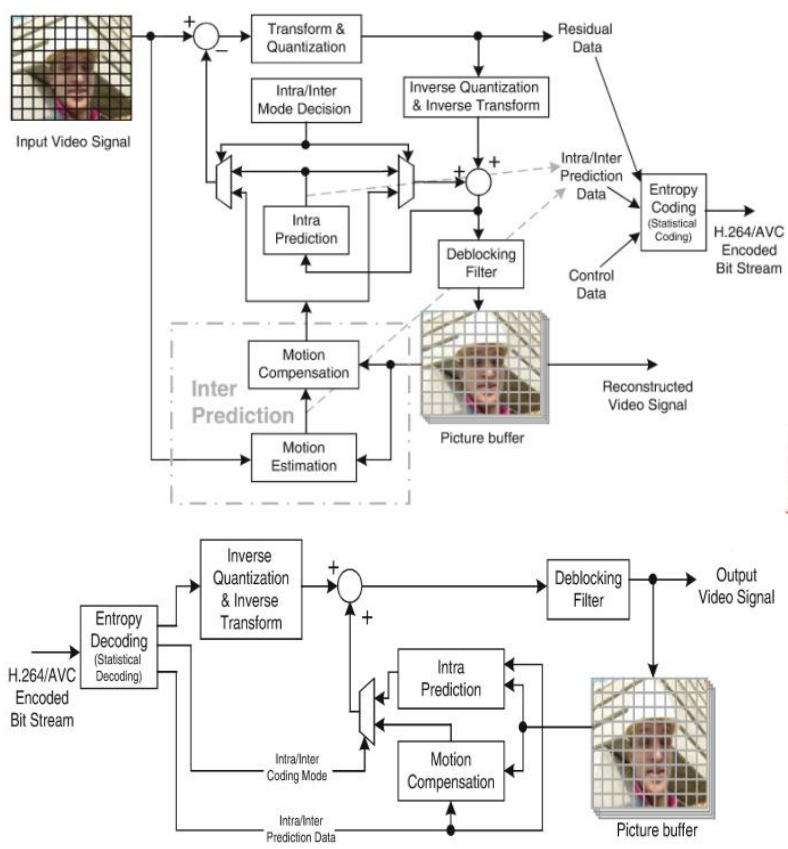

Fig: 1 Typical H.264 Encoder/Decoder [1]

In this paper, an implementation of Entropy decoder of Baseline profile is presented according to standard, which means to implement Exponential Golomb and CAVLC in Matlab and in Simulink and propose a method to implement the Entropy encoder in Matlab and Simulink as the encoder is not specified in the standard. In the next section the exponential Golomb design and implementation will be described in detail.

\section{EXPONENTIAL GOLOMB PRINCIPLES}

Exponential -Golomb codes are binary codes with variable length constructed according to a regular pattern.ExpGolomb supported by H.264 standard for encoding almost all syntax elements except those related to quantized transform coefficients [3][4].Variable length codes such as ExpGolomb is considered an efficient way of representing syntax elements by assigning short code words to frequently occurring syntax elements and long code words to less common syntax elements.[5]. 
Table 1 shows the first few Exp-Golomb code words that indexed by code Numbers. Also fromTable 1 it is noticed that these codes have a regular logical construction. So it can be encoded and decoded logically without the need for look-up table. An Exp Golomb codeword has the following structure:

[M Zeros Prefix] [1] [INFO]

(0)

Table1. Exp-Golomb Code words

\begin{tabular}{|c|c|}
\hline Bit string form & Range of code Num \\
\hline 1 & 0 \\
\hline $01 \mathrm{X}_{0}$ & $1 \ldots 2$ \\
\hline $\begin{array}{lllll}0 & 0 & 1 & X_{1} & X_{0}\end{array}$ & $3 \ldots 6$ \\
\hline $\begin{array}{lllllll}0 & 0 & 0 & 1 & X_{2} & X_{1} & X_{0}\end{array}$ & $7 \ldots 14$ \\
\hline $\begin{array}{lllllllll}0 & 0 & 0 & 0 & 1 & X_{3} & X_{2} & X_{1} & X_{0}\end{array}$ & $15 \ldots 30$ \\
\hline $\begin{array}{llllllllllllll}0 & 0 & 0 & 0 & 0 & 1 & X_{4} & X_{3} & X_{2} & X_{1} & X_{0}\end{array}$ & $31 \ldots 62$ \\
\hline$\ldots$ & $\ldots$ \\
\hline
\end{tabular}

Each codeword has a prefix of $\mathrm{M}$ zeros followed by 1 which is followed by INFO. Accordingly it is M-bit field carrying information. The first codeword has no leading zero or trailing INFO. Code words 1 and 2 have a single-bit INFO field; code words 3-6 have a two-bit INFO field and so on. The length of each Exp-Golomb codeword is $(2 \mathrm{M}+1)$ bits and each codeword can be constructed by the encoder based on its index code num as following:

$\mathrm{M}=$ floor $(\log 2[\operatorname{code} \mathrm{Num}+1])$

$\mathrm{INFO}=$ code $\mathrm{Num}+1-2 \mathrm{M}$

A codeword can be decoded as follows:

1. Count number of zeros till reaches 1 this number is $\mathrm{M}$

2. Read M-bit INFO field.

3. Code $\mathrm{Num}=2 \mathrm{M}+\mathrm{INFO}-1$

Code Num can be derived from Syntax element by different ways according to syntax element type as the standard supports 4 syntax element types ( Unsigned(Ue), Signed(Se) , Mapping(Me), Truncated (Te)). Mapping of syntax element to code Num for each type shall be according to the following procedure:

1-For Unsigned direct mapping, code num = syntax element Used for macro block type, reference frame index and others.

2- For Truncated mapping: if the largest possible value of syntax element is 1 , then a single bit $b$ is sent where $\mathrm{b}=$ ! Code num, otherwise Ue mapping is used

3- Signed mapping, used for motion vector difference, delta QP and others. Syntax element is mapped to code num as in Table2.

Table2. Signed mapping

\begin{tabular}{|l|l|}
\hline Code Num & Syntax element value \\
\hline 0 & 0 \\
\hline 1 & 1 \\
\hline 2 & -1 \\
\hline
\end{tabular}

\begin{tabular}{|l|l|}
\hline 3 & 2 \\
\hline 4 & -2 \\
\hline 5 & 3 \\
\hline 6 & -3 \\
\hline $\mathrm{K}$ & $(-1) \mathrm{K}+1 \mathrm{Ceil}(\mathrm{K}+2)$ \\
\hline
\end{tabular}

4- Mapped symbols: syntax element is mapped to code num according to a table specified in the standard [1][2].

\subsection{Exponential Golomb Implementation and Verification}

At first the Exponential - Golomb is implemented as an mfile in Matlab. Fig 2 shows the flow chart of the exponentialGolomb encoder implemented by Matlab. First step in encoder is to define the type of the syntax element. If the syntax element type is unsigned, then code Num equal syntax element. If the syntax element type is signed, then code Num calculates as in table2. If the type is mapping, one uses certain tables defined in the standard to obtain Code Num from syntax element. After calculating Code Num, one calculates $\mathrm{M}$ and Info and uses them to obtain encoded bits. Note that the truncated syntax element type encoded as unsigned if syntax element has value more than 1.And if syntax element equal 1 only one bit will be sent. When the input is a syntax element from unsigned type with a value of 226 to the encoder, it outputs the following result:

Encoded bits $=000000011100011$

This result can be confirmed by simple processing of the input according to equations (0), (1), and (2):-

$1-\mathrm{M}=$ Floor $\left[\log _{2}[226+1]\right]=$ Floor 7.826538 . Then $\mathrm{M}=7$ zeros.

$2-\mathrm{INFO}=226+1-27=99 \quad$ then binary form of info $=1100011$.

3-I follow that the Encoded bits of 226 should be 000000011100011 .

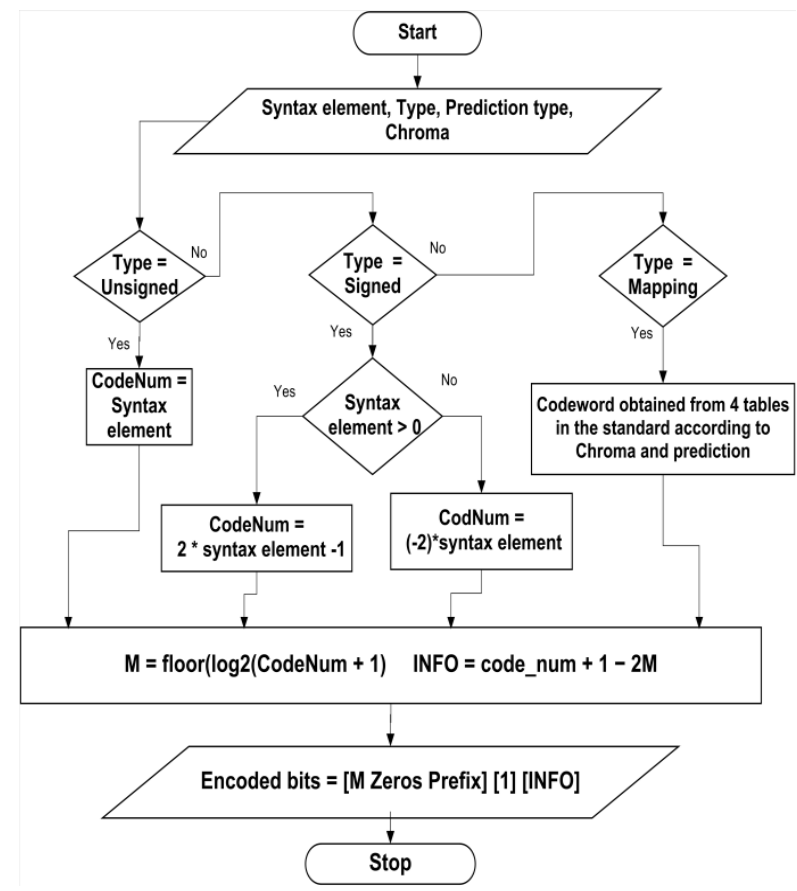

Fig 2: The flow chart for exponential Golomb encoder 
The decoder performs the inverse process of the encoder where it restores the original data form of decoded one. Fig 3 shows the flow chart of Exponential Golomb decoder implementation in Matlab. First step in decoder is to count the number of leading zeros of the received encoded bits to calculate M. Then calculate Info from the part after the first one. Then one uses $\mathrm{M}$ and Info to calculate code Num. One has to know the type of the received encoded bits to determine how to calculate syntax element from it. Note that the truncated syntax element type decoded as unsigned if syntax element has value more than 1.And if syntax element equal 1 only one bit will be received. When the input encoded bits are "000000011100011" and the syntax type is unsigned, the output will be a syntax element $=226$. To verify the correctness of the processing in the decoder the data is calculated according to the steps:

1-Count zeros before the first 1 , then $\mathrm{M}=7$

$2-\mathrm{INFO}=1100011$, which means in decimal INFO=99

3 - Syntax element $=2^{\wedge} 7+99-1=226$ which is the same output value from the encoder.

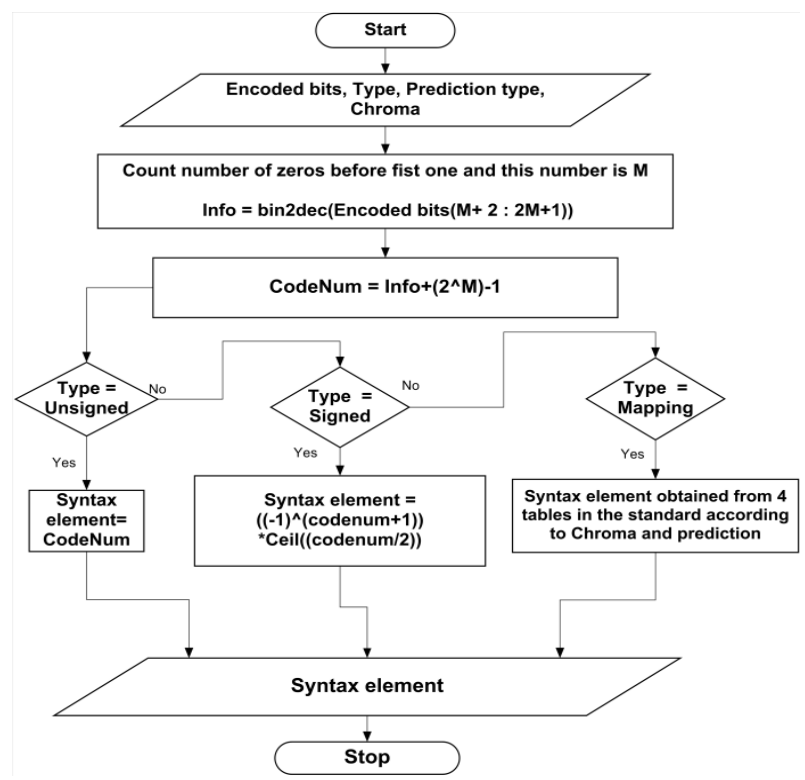

Fig 3: The flow chart of Exponential -Golomb decoder

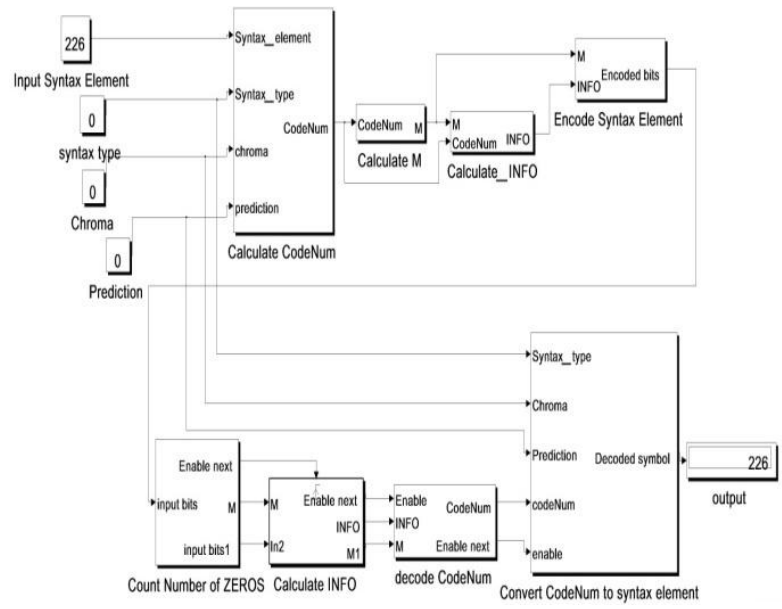

Fig 4: Simulink Exp-Golomb codec model
Fig 4 shows the Simulink model of the implemented ExpGolomb codec built according to the Matlab model developed in the previous section. The encoder takes syntax element and type, while in case of mapping type it also takes chroma and prediction, as input. The first step in encoder is to calculate Code Num then calculate $\mathrm{M}$ and Info then use them to figure out Encoded bits. The decoder takes encoded bits and type, while in case of mapping type it also takes chroma and prediction, as input. The first step is to count leading zeros to calculate $M$ then uses it to calculate Info and at last decodes Code Num then calculates syntax element according to syntax type. The model consists of the encoder and decoder in a loop back connection for the functional verification of the whole codec. It is clear from the figure that the error counter of the difference between the input and the output is equal to zero proving the correctness of the Simulink implementation

\section{CAVLC PRINCIPLE}

In this section Matlab implementation of the CAVLC will be carried out similar to Exp-Golomb codec. The Context adaptive variable length coding (CAVLC) is an Entropy coding method which is used to compress digital data by examining the frequency of patterns within it and representing frequently occurring patterns with smaller number of bits. It is used to encode quantized residual data of $4 \mathrm{X} 4$ or $2 \mathrm{X} 2$ blocks of transform coefficients. A residual block is the difference between the predicted and the input video data and is obtained after integer discrete cosine transform and quantization. In order to increase compression ratio, CAVLC in h.264 standard introduces the concept of context model to map the probability of symbols more accurately. However, to provide such a high compression ratio the computational complexity becomes higher because of search for code words in several look-up tables. CAVLC is designed to take advantage of several characteristics of quantized $4 \times 4$ blocks of transform coefficients such as:

- Existence of long runs of zeros after zigzag scanning.

-Highest number of non-zero coefficients after zigzag scanning are often sequences of $+/-1$.

-The number of non-zero coefficients in neighboring blocks is correlated.

-Level of non-zero coefficients is usually higher at the start of the reordered array near the DC coefficient, and lower towards the higher frequencies.

Before encode with CAVLC the residual data must be reordered using ZIGZAG method, as in depicted Fig5, then CAVLC steps take place. CAVLC coding consist of five steps as shown in Fig 6 [2] [6][7].

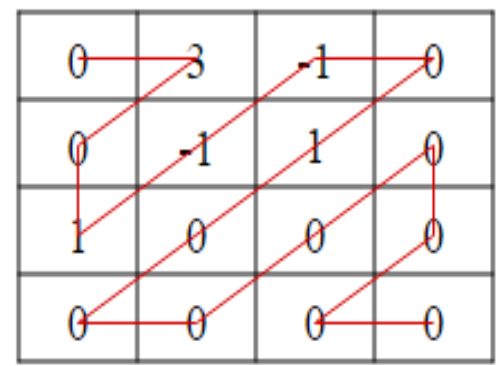

Fig 5: ZigZag reordering of $4 \times 4$ residual transform matrix 


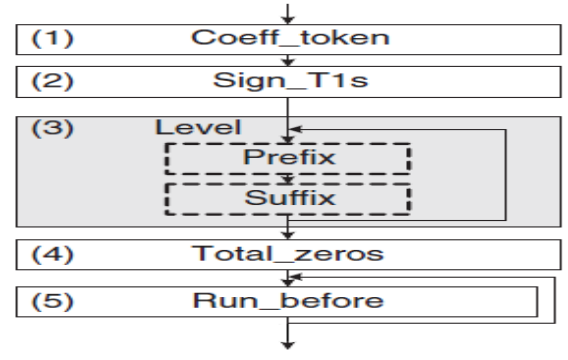

Fig 6: CAVLC coding flow chart

1-Coeff_token: This step encodes the number of nonzero coefficients (TNZ) and the number of trailing ones (T1). TNZ can be anything from 0 to 16 and T1 can take values from 0 to 3 . If there are more than three trailing $+/-1 \mathrm{~s}$, only the last three are treated as 'special cases' and any others are coded as normal coefficients. There are four choices of lookup table to use for encoding Coeff_token, three variablelength code tables and a fixed-length code table. The choice of table depends on the number of non-zero coefficients in the left and upper previously coded blocks, nA and $\mathrm{nB}$ respectively [8]

- If $\mathrm{nA}, \mathrm{nB}>0$ then $\mathrm{nC}=\mathrm{nA}+\mathrm{nB}>>1$,

- Else if only $\mathrm{nB}=0$ then $\mathrm{nC}=\mathrm{nA}$,

- Else if only $\mathrm{nA}=0$ then $\mathrm{nC}=\mathrm{nB}$,

- $\quad$ Else $\mathrm{nC}=0$

After calculating $\mathrm{nC}$ parameter, then choose one of look-up tables for encoding according to Table3.

Table 3. Choice of look-up table for Coeff_token

\begin{tabular}{|l|l|}
\hline $\mathbf{n C}$ & Tables for Coeff_token \\
\hline 0,1 & VLC table 1 \\
\hline 2,3 & VLC table 2 \\
\hline $4,5,6,7$ & VLC table 3 \\
\hline 8 or above & FLC, fixed length coding \\
\hline
\end{tabular}

2- Sign of trailing ones: One bit to code the sign of each trailing ones, 0 for positive sign, and 1 for negative sign.

3- Levels: In this step encode the sign and magnitude of the remaining nonzero coefficients in reverse order level composed of two parts, level_prefix and level_suffix. Level_prefix is a b leading zeros followed by 1 according to Table4.

Table4. Codeword table for level_prefix

\begin{tabular}{|l|l|}
\hline level_prefix & bit string \\
\hline 0 & 1 \\
\hline 1 & 01 \\
\hline 2 & 001 \\
\hline 3 & 0001 \\
\hline 4 & 00001 \\
\hline 5 & 000001 \\
\hline
\end{tabular}

\begin{tabular}{|l|l|}
\hline 6 & 0000001 \\
\hline 7 & 00000001 \\
\hline$\cdots$ & $\cdots \cdots$ \\
\hline
\end{tabular}

Level_suffix is an integer code of size suffix length. Largemagnitude coefficients have low probability, so, its suffix length is large. Small-magnitude coefficients have high probability, so, its suffix length is small. The choice of suffix Length I subjected to the following rules:

Initial suffix Length $=0$, except if $((\mathrm{TNZ}>10)$ and $(\mathrm{T} 1<3))$ then initial suffix length $=1$.

If the magnitude of this coefficient is larger than a threshold, increment suffix Length, up to a maximum suffix Length $=6$ according to table5.

4- Total zeros: the sum of all zeros before each non zero coefficient it coded according to look up table given by the standards.

5- Run before: the number of run zeros preceding each nonzero level in reverse zigzag order.

Table5. Thresholds for determining whether to increment suffix_length

\begin{tabular}{|l|l|}
\hline $\begin{array}{l}\text { Current suffix } \\
\text { length }\end{array}$ & $\begin{array}{l}\text { Threshold to increment suffix } \\
\text { length }\end{array}$ \\
\hline 0 & 0 \\
\hline 1 & 3 \\
\hline 2 & 6 \\
\hline 3 & 12 \\
\hline 4 & 24 \\
\hline 5 & 48 \\
\hline 6 & highest value reached \\
\hline
\end{tabular}

\subsection{Matlab CAVLC Encoder/Decoder Implementation}

At first the Context adaptive variable length (CAVLC) is implemented as m-file in Matlab. Fig 7 shows the flow chart of CAVLC encoder. The Encoder takes syntax elements, nA and $\mathrm{nC}$ as inputs. The first step is to reorder syntax elements by ZigZag method. Then count the number of trailing ones and determine the sign of each trailing ones. Then the encoder calculates $\mathrm{nC}$ to define the look up table which is used to obtain the Coeff_token according to the number of trailing ones and total number of non zero coefficients (TNZ). The next step is for- loop repeated by TNZ-trailing ones to calculate level_prefix and level_suffix for every non zero coefficient in order to calculate level_code. Finally, it encodes the number of total zeros and each run of zeros before each non zero element. 


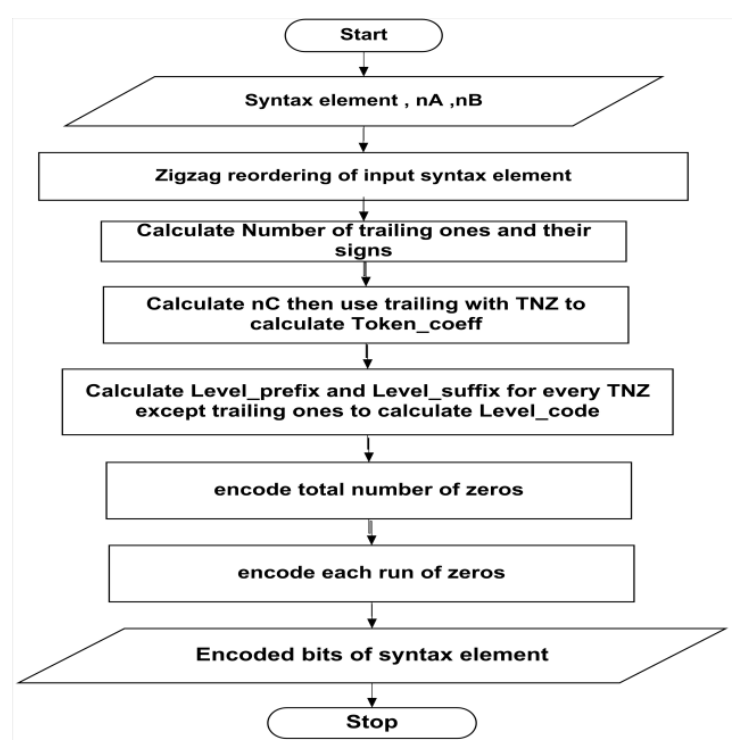

Fig 7: The flow chart for CAVLC encoder

The decoder performs the inverse processes of the decoder, where it restores the original form of the data. Fig 8 shows the flow chart of Context adaptive variable length (CAVLC) decoder implementation in Matlab. The Decoder takes encoded bits, $\mathrm{nA}$ and $\mathrm{nC}$ as inputs. The first step is to calculate $\mathrm{nC}$ to determine which look up table will be used in calculating Total number of coefficient and number of trailing ones. After that, the decoder calculates the sign of each trailing ones according to that 0 means positive sign and 1 means negative sign. The next step is to decode Level and sign for each total non zero coefficient. Then decode of the total number of zeros and each run of zeros before every TNZ. The last step is to reorder the data into 4X4 block

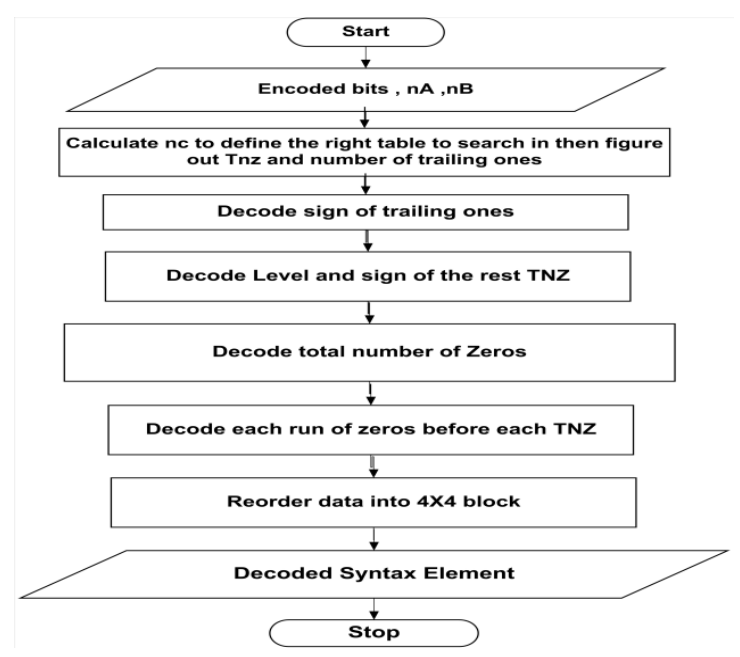

Fig 8: The flow chart for CAVLC decoder

\subsubsection{Encoder Implementation}

Table6. CAVLC encoder example

\begin{tabular}{|c|c|c|}
\hline Element & Value & Code \\
\hline Coeff_token & $\begin{array}{c}\text { Total_Coeff = 5, T1s } \\
=3 \text { (use Num VLC0) }\end{array}$ & 0000100 \\
\hline T1 sign (4) & + & 0 \\
\hline T1 sign (3) & - & 1 \\
\hline T1 sign (2) & - & 1 \\
\hline
\end{tabular}

\begin{tabular}{|c|c|c|}
\hline Level (1) & $\begin{array}{c}+1 \text { (level prefix }=1 \text { at } \\
\text { suffix Length }=0)\end{array}$ & 1 \\
\hline Level (0) & $\begin{array}{c}+3(\text { level prefix }=001 \\
\text { at suffix length }=1)\end{array}$ & 0010 \\
\hline Total Zeros & 3 & 111 \\
\hline run before(4) & $\begin{array}{c}\text { Zeros Left }=3 ; \text { run } \\
\text { before }=1\end{array}$ & 10 \\
\hline run before(3) & $\begin{array}{c}\text { Zeros Left }=2 ; \text { run } \\
\text { before }=0\end{array}$ & 1 \\
\hline run before(2) & $\begin{array}{c}\text { Zeros Left }=2 ; \text { run } \\
\text { before }=0\end{array}$ & 1 \\
\hline run before(1) & $\begin{array}{c}\text { Zeros Left }=2 ; \text { run } \\
\text { before }=1\end{array}$ & 01 \\
\hline run before $(0)$ & $\begin{array}{c}\text { Zeros Left }=1 ; \text { run } \\
\text { before }=1\end{array}$ & $\begin{array}{l}\text { No code required; } \\
\text { last coefficient. }\end{array}$ \\
\hline $\begin{array}{l}\text { The output } \\
\text { code word }\end{array}$ & 0000100011100 & 0111101101 \\
\hline
\end{tabular}

Here the encoding process will be demonstrated by an example. The data to be encoded is given in fig 5 where it is reordered by zigzag scanning resulting in the data series in the form of $(0,3,0,1,-1,-1,0,1,0,0,0,0,0,0,0,0)$. The encoding process is carried out according to the algorithm given previously in fig 7 . According to the first step in the algorithm, the Total_coeff $=5, \mathrm{~T} 1=3$ and $\mathrm{nC}$ is taken zero as there is no previous encoded blocks. So, from table 3 these values result in choosing (VLC table1).Two approaches are used to obtain the VLC of Total_coeff and T1.

The first approach is to use the same order of the standard as in Table 7 and calculate the required row number by this equation:

If (Total_coeff >2) then row number=4(Total_coeff-3) $+\mathrm{T} 1+7$,

Else if $($ Total_coeff $==2$ ), then row number $=\mathrm{T} 1+4$,

Else if (Total_coeff $==1$ ), then row number $=\mathrm{T} 1+2$,

Else row number $=1$

Table7: Coeff_token mapping to Total_coeff and trailing ones

\begin{tabular}{|c|c|c|c|}
\hline Row number & T1 & Total_Coeff & $\mathbf{0} \leq \mathbf{n C}<\mathbf{2}$ \\
\hline 1 & 0 & 0 & 1 \\
\hline 2 & 0 & 1 & 000101 \\
\hline 3 & 1 & 1 & 01 \\
\hline 4 & 0 & 2 & 00000111 \\
\hline 5 & 1 & 2 & 000100 \\
\hline 6 & 2 & 2 & 001 \\
\hline 7 & 0 & 3 & 000000111 \\
\hline 8 & 1 & 3 & 00000110 \\
\hline 9 & 2 & 3 & 0000101 \\
\hline 10 & 3 & 3 & 00011 \\
\hline$\cdots$ & $\cdots$ & $\cdots$ & $\cdots$ \\
\hline
\end{tabular}


The second approach is to reorder the table of the standard to make 2D table with 16 rows representing the Total_coeff and 4 columns representing $\mathrm{T} 1$ as shown in table 8 .

Table8: Method to reorder Coeff_token mapping to Total_coeff and trialing ones table.

T1

\begin{tabular}{|c|c|c|c|c|}
\hline & 0 & 1 & 2 & 3 \\
\hline 0 & 1 & - & - & - \\
\hline 1 & 000101 & 01 & & 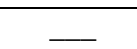 \\
\hline 2 & 00000111 & 000100 & 001 & \\
\hline 3 & $\begin{array}{c}00000011 \\
1\end{array}$ & 00000110 & 0000101 & 00011 \\
\hline$\ldots$ & $\ldots$ & $\ldots$ & $\ldots$ & $\ldots$ \\
\hline 1 & 00000000 & 00000000 & 00000000 & 00000000 \\
\hline 5 & 00000111 & 00001010 & 00001001 & 00001100 \\
\hline 1 & 00000000 & 00000000 & 00000000 & 00000000 \\
\hline 6 & 00000100 & 00000110 & 00000101 & 00001000 \\
\hline
\end{tabular}

The second Step in the encoding process applied on the example: here there are three trailing ones with sign,,+-- so the encoded symbol will be 011 as given in Table 6 .

The third Step in the encoding process: this step encodes the remaining non zero coefficients +3 and +1 . Each coefficient is transformed to level code by transforming positive coefficient to even level code and negative coefficient to odd level code according to the following rules:-

Levelcode $=2 *$ Coefficient -2 for positive coefficient,

Levelcode $=-2 *$ Coefficient -2 for negative coefficient,

If ((index of coefficient $==$ number of trailing ones) \& (number of trailing ones $<3$ ) then,

\section{Levelcode $=$ Levelcode -2}

Then each level code encoded has two parts, a level_prefix which is determined as in table 4 and a level_suffix which is determined according to the following rules:

Initial suffix_length is calculated as in section 4 and incremented as in table 5 .

If (level_code binary right shifted by suffix_length) $<14$,

Then level_prefix $=$ (level_code binary right shifted by suffix_length),

And Levelsuffixsize =suffix_length,

Else if $(($ level code<30) \& (suffix_length==0)),

Then level_prefix $=14$ and Levelsuffixsize $=4$,

Else if ((suffix_length>0) \& (level_code binary right shifted by suffix_length==14),

Then level_prefix $=14$ and Levelsuffixsize=suffix_length,

Else level_prefix $=15$ and Levelsuffixsize $=12$ (as in baseline profile level_prefix maximum value is 15).

Let us apply the above rules in this example. The first non zero coefficient is +1 . The level code for that coefficient $=0$ and the initial suffix_length $=0$. So level_prefix is equal 1 from table 4 and Level_suffixsize $=0$.Then the codeword for +1 will be 1 . Similarly, the codeword of +3 is 0010 as in table 6 .

The fourth Step for encoding in this example: the total non zero elements is equal 5 and the total zeros before these elements is equal 3. From the look up table in the standards, the code word will be ' 111 '.

The fifth Step for encoding: in this step the encoder encodes the number of zeros before each non zero element. In table 6 , the number of non zero elements equals 5 , so as shown for each element, there is a code word except for the last one as the total zeros are encoded before. This code word is calculated from a the look up table in the standard. For each code word, the number of zeros before the non zero coefficient and the number of zeros left are needed. For example the first non zero coefficient has 1 zero run before and the zeros left $=3$ so from the look up table its code word is 10 and so on for the remaining run before.

\subsubsection{Decoder Implementation}

Decoder must restore the original data from the encoded data according to flow chart in fig 8 . As has been done with the encoder, the decoder will be implemented in this section on the light of an example, where the above encoded sequence will be the input to the decoder and transformed back to its original form so the input to the decoder is 000010001110010111101101.

Table 9: CAVLC Decoder example

\begin{tabular}{|c|c|c|c|}
\hline Code & Element & Value & $\begin{array}{l}\text { Output } \\
\text { array }\end{array}$ \\
\hline 0000100 & Coeff_token & $\begin{array}{c}\text { TotalCoeffs }=5, \mathrm{~T} 1 \mathrm{~s}= \\
3\end{array}$ & Empty \\
\hline 0 & T1 sign & + & 1 \\
\hline 1 & T1 sign & - & $-1,1$ \\
\hline 1 & T1 sign & - & $\begin{array}{c}-1,-1 \\
1\end{array}$ \\
\hline 1 & Level & $\begin{array}{c}+1 \text { (suffix_Length=0 } \\
\text { increment } \\
\text { suffix_Length) }\end{array}$ & $\begin{array}{l}1,-1, \\
-1,1\end{array}$ \\
\hline 0010 & Level & +3 (suffix_Length $=$ & $\begin{array}{c}3,1,-1, \\
-1,1\end{array}$ \\
\hline 111 & Total Zeros & 3 & $\begin{array}{c}3,1,-1, \\
-1,1\end{array}$ \\
\hline 10 & run before & 1 & $\begin{array}{l}3,1,-1, \\
-1,0,1\end{array}$ \\
\hline 1 & run before & 0 & $\begin{array}{l}3,1,-1, \\
-1,0,1\end{array}$ \\
\hline 1 & run before & 0 & $\begin{array}{l}3,1,-1, \\
-1,0,1\end{array}$ \\
\hline 01 & run before & 1 & $\begin{array}{c}3,0,1 \\
-1,-1 \\
0,1\end{array}$ \\
\hline
\end{tabular}

First Step: As shown in Table 9 first the decoder decodes the TotalCoeffs and T1. This process takes long time, as in the standard, for the VLC table with $4 \mathrm{X} 4$ block there are 62 
elements in the lookup table to be scanned to figure out the TotalCoeffs and T1.So, it is tried here to subdivide this large look up table into smaller ones according to the number of leading zeros as shown in fig 9. Then one has to count the number of leading zeros of the received stream and choose the appropriate sub lookup table for it this will reduce the number of access times of the lookup tables .Here using the 62 element look up table needs 18 access trials to find a match. While with the use of these smaller look up tables the access trial will be 3 times. The code word 0000100 means that the total non zero coefficient $=5$ and the number of trailing ones $=3$.

The second Step: from previous step the number of trailing ones is 3 so the decoder takes the next 3 bits to determine their signs.

\begin{tabular}{|c|c|}
\hline \multirow{3}{*}{ 疍 } & 1 \\
\hline & 000101 \\
\hline & 01 \\
\hline ? & 00000111 \\
\hline$\frac{4}{8}$ & 000100 \\
\hline$\vec{E}$ & \\
\hline 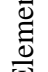 & $\begin{array}{l}000000000000110 \\
0000000000000101\end{array}$ \\
\hline तु & 0000000000001000 \\
\hline
\end{tabular}

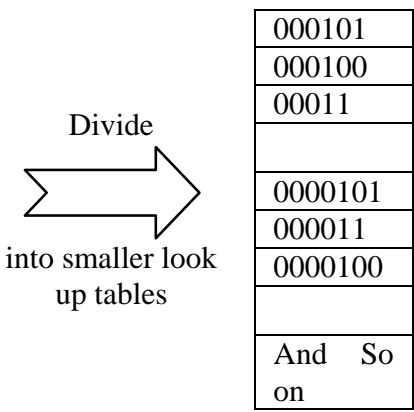

Fig9: The large look up table for the Coeff_token is divided into sub lookup tables according to the number of leading zeros

Third Step: from step one the number of non zero coefficients and the number of trailing ones are known. Then, the difference specifies the number of times to repeat this step to obtain the value and the sign of the remaining non zero coefficient. Here the decoder count the no of zeros to the known level_prefix then Levelsuffixsize is determined according to the following conditions:

If ( (level_prefix==14)\&(suffix_length==0)

Then Levelsuffixsize $=4$,

Else if (level_prefix==15),

Then Levelsuffixsize $=12$

Else Levelsuffixsize $=$ suffix_length.

After that the decoder converts the levelcode to the non zero coefficient by the following rules:

Coefficient $=($ levelcode +2$)>>1$ for even levelcode

Coefficient $=($-levelcode- 1$)>>1$ for odd levelcode .

As shown in table 9 (the Total non zero coefficient - the trailing ones =2) so There are two non zero coefficient to be decoded. The codeword of the first coefficient is ' 1 ' and from table 4 it has level_prefix $=0$ and it has no level_suffix, then the decoded levelcode $=0$ and the decoded coefficient $=+1$. By using the above rules once again, the codeword of the second coefficient ' 0010 ' is decoded as +3 .

The fourth Step in decoding: from step one, the total number of non zero coefficients(TNZ) is 5 , which means have to search in column number 5 in the look up table proposed by the standard to decode the total number of zeros. Here in this example the required code word '111' and that means total zeros before non zero elements equal 3 as in table 9 .
The fifth Step in the decoding: from step one TNZ $=5$, so the decoder has to decode the run before zeros to each coefficient. There is a look up table in the standard defining the code word of the run before zeros according to number of zeros left and number of run before zeros. In this example, at first the decoder put zero left $=$ total number of zero which decoded in step number four then zeros left $=3$. This means that searching for a match from column number 3 in the look up table. Here the decoder find a match for code word '10' which means that before element number five there is one zero. By the same procedure '1', '1' ,'01' means, before element number four there is no zeros, and before element number three there is no zeros, while before element number two there is one zero. The run before zeros before the element number one did not have to be encoded because the number of zero left is known from total zero which here is one.

\subsection{Cavlc Encoder/ Decoder Simulink Implementation}

Fig 10 shows the Simulink model of the implemented CAVLC encoder. The encoder takes Syntax element 4X4 blocks, $\mathrm{nA}$ and $\mathrm{nB}$ as inputs and run all steps as has been done in the Matlab implementation. So, the first process in the encoder is to reorder the input syntax by ZigZag method then the first, second, fourth and fifth steps take place at the same time as all these steps not depend on each other. The third step and the final stage are enabled by the stage of step 1 and 2 block. To encode the remaining non zero coefficients and after some delay, the first step enables the final stage which reorder all the outputs from each stage and output the encoded bits which represent the encoded syntax.

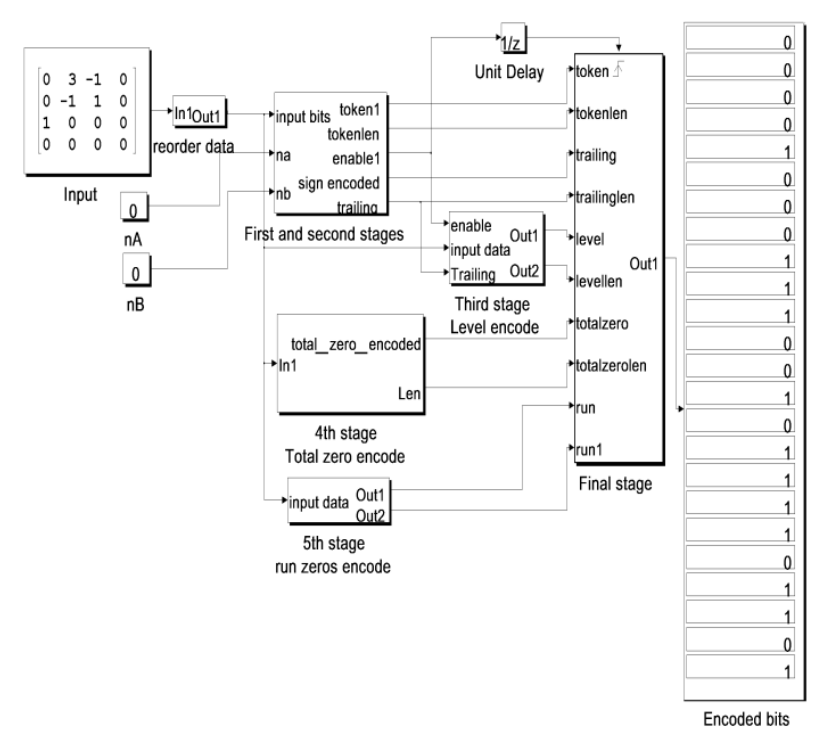

Fig 10: Simulink CAVLC encoder model

Fig 11 shows the Simulink model of the implemented CAVLC decoder where the processing is carried out in stages similar to the Matlab decoder model. The decoder takes encoded bits, $\mathrm{nA}$ and $\mathrm{nB}$ as inputs. First step in decoder is to decode token coefficient to calculate TNZ and trailing ones. Two stages can proceed at the same time, namely the second and third stage. The second stage is to figure out the sign of each trailing ones. The third stage is to decode the level and sign of each non zero coefficient and store these levels in registers. Then, the fourth stage can proceed to 
decode the total number of zeros. Finally, stage five collect all outputs from the previous stages and from registers and decode run before zeros and then reorder all outputs in $4 \mathrm{X} 4$ block

Fig 12 shows the encoder and decoder of CAVLC in a loop back connection for the functional verification of the whole codec. It is clear from the figure that the error counter of the difference between the input and the output is equal to zero proving the correctness of the Simulink implementation.

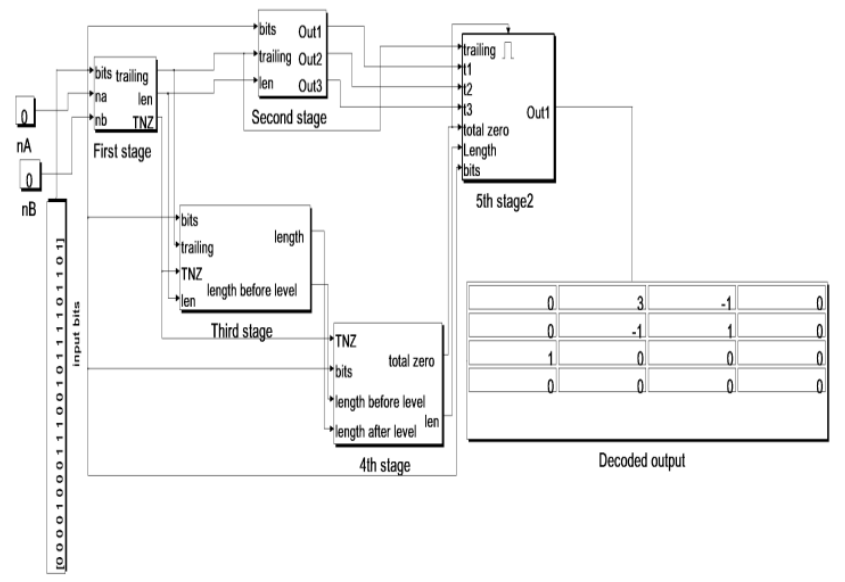

Fig 11: Simulink CAVLC decoder model

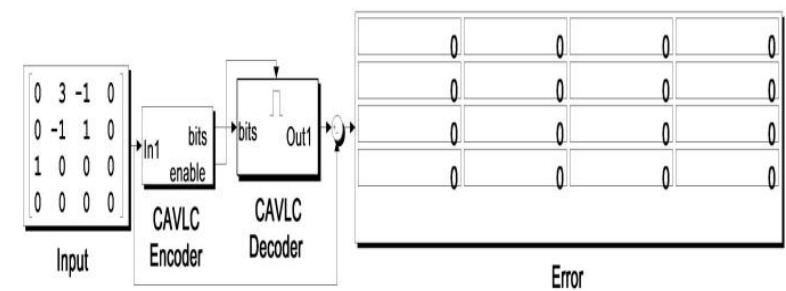

Fig 12: CAVLC codec loop back for verification

Similarly, the CAVLC encoder, decoder and loopback verification for $2 \mathrm{X} 2$ residual blocks are implemented depicted in Figs 13, 14 and 15, respectively.

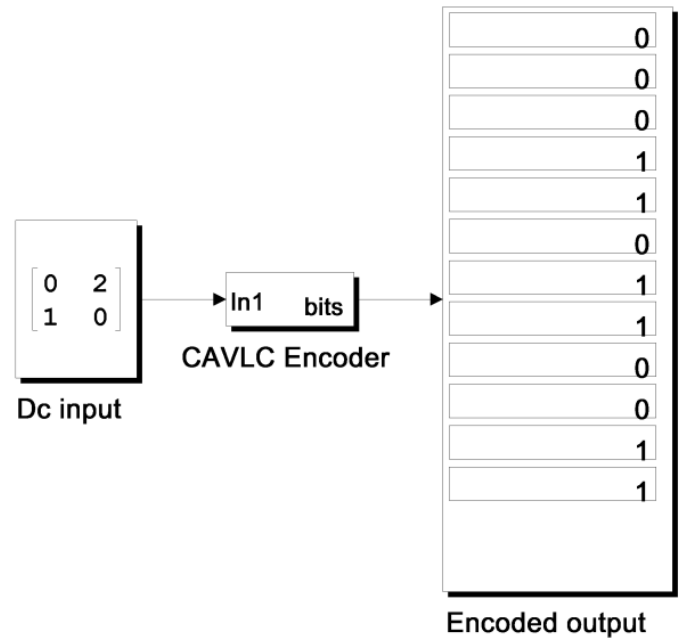

Fig 13: CAVLC 2X2 Simulink encoder

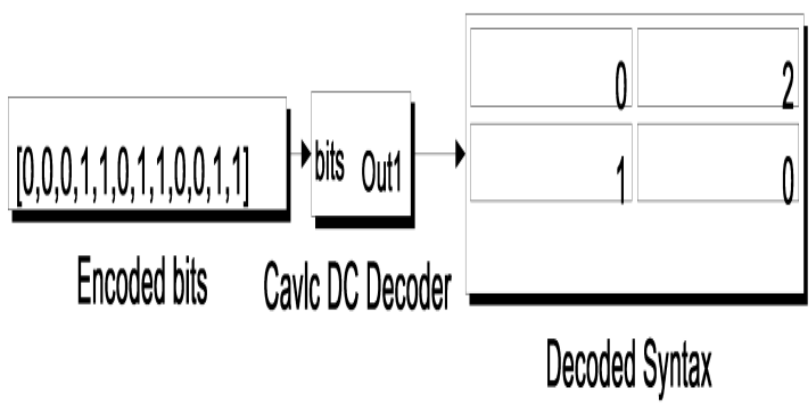

Fig 14: CAVLC 2X2 Simulink decoder

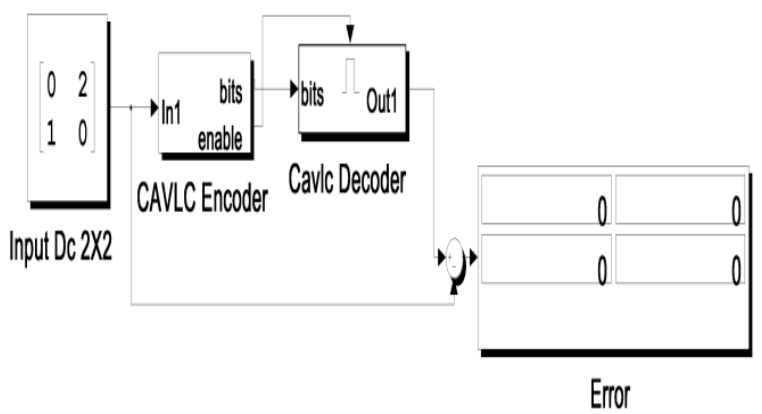

Fig 15: CAVLC 2X2 codec loopback for verification

\section{CONCLUSION}

In this paper the implementation of encoders and decoders of the Exponential Golomb and the CAVLC codec techniques are carried out according to the standards of the video codec H264 by Matlab and Simulink. This implementation is a primary step towards VHDL implementation on FPGA. The theoretical basis for the implementation is first described and then appropriate algorithms of the codec are presented. Matlab m-files are produced for all the encoders and decoders. Then Simulink model is built for both codec. The validation of the implementation is verified by straight forward calculations and also by loopback method. This project is a part of custom implementation of H264 video codec.

Future work is to develop a technique to reduce the time of searching tables in the decoder to reduce the time taken by the decoder to decode a symbol. Implement encoder and decoder of Exponential Golomb and CAVLC using VHDL on FPGA.

\section{ACKNOWLEDGEMENT}

The article is partially supported by a grant of the Foundation of Computer Science, NY, USA vide FCS/RT56/15"

\section{REFERENCES}

[1] ITU-T Recommendation H.264, 06-2011.

[2] Iain E.RICHARDSON, The H.264 Advanced Video Compression Standard, Second edition , 2010, John Wiley \& Sons.

[3] Jorn Ostermann, Jan Bormans, Peter List,Detlev Marpe, Matthias Narroschke, Fernando Pereira, Thomas Stockhammer, and Thomas Wedi,"Video coding with H.264/AVC:Tools, Performance, and Complexity", FIRST QUARTER 2004, IEEE CIRCUITS AND SYSTEMS MAGAZINE 
[4] Detlev Marpe and Thomas Wiegand, Heinrich Hertz Institute (HHI),Gary J. Sullivan, Microsoft Corporation," The H.264/MPEG4 Advanced Video Coding Standard and its Applications", August 2006, IEEE Communications Magazine

[5] Iain E. Rechardson, "H.264 and MPEG-4 Video Compression", 2003, John Wiley \& Sons.

[6] ThomasWiegand, Gary J. Sullivan, Senior Member, IEEE, Gisle Bjøntegaard, and Ajay Luthra, Senior Member, IEEE, "Overview of the H.264/AVC Video Coding Standard", IEEE TRANSACTIONS ON
CIRCUITS AND SYSTEMS FOR VIDEO TECHNOLOGY, VOL. 13, NO. 7, JULY 2003

[7] Xiaohua Tian, Thinh M. Le, Yong Lian, Entropy Coders of the H.264/AVC Standard

[8] Algorithms and VLSI Architectures, 2011, SpringerA HIGH PERFORMANCE AND LOW POWER HARDWARE ARCHITECTURE FOR H.264 CAVLC ALGORITHM, Esra Sahin and Ilker Hamzaoglu Faculty of Engineering and Natural Sciences, Sabanci University 34956, Orhanli, Tuzla, Istanbul, TURKEY. 